Piotr Kubasiak

Universität Wien

\title{
Kraft der Bilder. Zur Rolle der Metapher bei F. Nietzsche und K. Michalski und ibre Implikationen für die Theologie
}

\section{Abstract}

Powerful Images. On the Significance of the Metaphor for F. Nietzsche and K. Michalski and its Implications FOR THEOLOGY

The crisis of the theological language has been a subject of heated debate for a long time. Johann Baptist Metz even stated that the "Gotteskrise" (crisis of God) is in the first place a crisis of language. However, the identification of a new and appropriate language is not an easy task and - as the History of the Church shows - it is always a work of several generations. The following text refers to the importance of metaphors for theological language. A metaphor is understood not only as a literary form, but also as a method which next to understanding touches on emotions and the imagination giving it a special persuasive power. It should not replace the reflective language of theology, but complement it. The Polish philosopher Krzysztof Michalski discovered in the work of Nietzsche the idea that a metaphor on the one hand has a concrete effect, but on the other hand shows that the most important thoughts can only be expressed in this form. Simple terms can never encompass all of life, there is always a greater sense to them than the mere words suggest. Therefore, metaphors in Nietzsche's texts become metadefinitions, which always refer to something else, to something larger, to the limit of our knowledge. 
Even though the metaphor will not solve the problem of the crisis of language in theology, it points to an important aspect of religious speech. It is a suitable - though not the only one - means of reflection on the Revelation, which ultimately does not only provide information about God, but aims at our life and wants to lead us to a communion with God.

\section{Keywords}

theological language, metaphor, Gotteskrise, Friedrich Nietzsche, Krzysztof Michalski

\section{EINLEITUNG}

Seit den neunziger Jahren wird das von Johann Baptist Metz geprägte Stichwort „Gotteskrise“ viel diskutiert. Metz definiert jedoch jene Gotteskrise nicht. Er nennt nur zwei Phänomene der Immunisierung gegenüber dieser Gotteskrise. Die theologische Immunisierung stellt für Metz die strikte Offenbarungstheologie dar. Die Differenz zwischen der von uns erfahrenen Welt und der Offenbarungswelt, zwischen Natur und Gnade ist nicht mehr sichtbar. Es scheint ein Offenbarungs- und Gnadenabsolutismus zu herrschen. Der Mensch, der verunsicherte, der zweifelnde und ungläubige findet hier keinen Platz. Die zweite, kirchliche Immunisierung besteht für Metz in der Verkirchlichung der Gottesrede. Das II. Vaticanum spricht nicht mehr wie das Erste von Gott, sondern von einem durch die Kirche verkündeten Gott. Metz führt dieser Befund zu zwei Rückfragen: Sprechen die Kirchen eine für die Gotteskrise empfindliche Sprache? Und: Gott kommt nicht mehr zur Sprache, aber kommen wir noch zur Sprache? Anhand dieser Fragen stellt er fest: „Die Gotteskrise ist zunächst einmal eine Krise der Gottessprache“2.

Für Tiemo Rainers Peters ist Gotteskrise „die Krise einer hochabstrakten Gottesprädikation in Theologie und Verkündigung (... “3.

I Vgl. J. B. Metz, Kirche in der Gotteskrise, in: C. Amery (Hrsg.), Sind die Kirchen am Ende?, Regensburg 1995, S. I63.

2 J. B. Metz, Kirche in der Gotteskrise, S. I6I.

3 T. R. Peters, Mebr als das Ganze. Nachdenken über Gott an den Grenzen der Moderne, Ostfildern 2008, S. 75-76. 
Sie sei gleichzeitig eine Krise der Kirche und der Theologie, die als Selbstzweck dienen, die auf die Fragen des Menschen „nur noch mit verbrauchten Geheimnissen“ antworten. Es handelt sich um eine Krise des Gottesbegriffs. ${ }^{4}$

In die gleiche Richtung geht auf dem Feld der praktischen Theologie Fritz Weidmann, der die Krise der religiösen Sprachen in einigen Erscheinungen sieht:

Als Symptome hierfür lassen sich ein unwahres und klischeehaftes Vokabular, eine dominierend theologisch-dogmatische Begrifflichkeit, ein mangelnder Wirklichkeitsbezug, eine fehlende Orientierung des Wortschatzes am gesellschaftlich-kulturellen Wandel, ethisch-moralische Leerformeln, eine kitschig-sentimentale Sprachgestalt und manchmal auch eine Anbiederung in Form einer künstlich hergestellten Modernität in der Sprache der kirchlichen Verkündigung überhaupt wie auch des Religionsunterrichts ausmachen. ${ }^{5}$

Die Tatsache, dass mit der theologischen und kirchlichen Sprache etwas nicht stimmt, ist bereits lange bekannt. Wie aber die theologische Forschung zeigt, es ist gar nicht so einfach, eine neue Sprache zu finden, die die alte ersetzen könnte. Die Kirchengeschichte zeugt davon, dass eine angemessene, präzise und verständliche Sprache ein Werk mehrerer Generationen ist, das eigentlich nie vollendet werden kann. Im vorliegenden Text soll daher nur auf einen Aspekt der Sprache hingewiesen werden, nämlich auf das Phänomen der Metapher, das als ein angemessenes Mittel zum Ausdruck der wichtigsten Gedanken dienen kann.

\section{Metapher in der Theologie}

Ob in der Bibel, bei den Kirchenvätern, der Scholastik oder neueren Theologierichtungen spielt die Metapher eine bedeutende Rolle. Unter dem Begriff der Metapher versteht man jedoch nicht nur eine literarische Form, sondern auch eine Methode, die die Wirklichkeit nicht

4 Vgl. T. R. Peters, Mehr als das Ganze, S. 76-77.

5 Vgl. F. Weidmann, Sprache und Religionsunterricht, in: F. Weidmann (Hrsg.), Didaktik des Religionsunterrichts. Ein Leitfaden, Donauwörth 19977, S. I66. 
präzise zu beschreiben versucht, sondern im Kopf des Autors und des Empfängers die gleiche Vorstellung bzw. das gleiche Objekt hervorruft. Bei der Metapher handelt es sich nicht nur um ein intellektuelles Ergebnis, sondern sie spricht auch die Gefühle und die Vorstellungen an. Sie will nicht nur die Ansichten verändern, sondern auch zu einem Umdenken bzw. Bekehren führen. Die Metapher scheint für die Theologie, die nichts anderes als die Reflexion der Offenbarung ist, besonders geeignet zu sein. Die Offenbarung ist erstens in menschlicher Sprache ausgedrückt und, was damit zusammengeht, sie ist nicht immer präzise und eindeutig. Des Weiteren will die Offenbarung nicht nur Informationen liefern, sondern vor allem zur Veränderung des Lebens führen. Mit dem intellektuellen Engagement muss also immer ein existenzielles Engagement zusammengehen. ${ }^{6}$

Metaphorische Sprache darf natürlich nicht die Reflexionssprache der Theologie ersetzen. Sie muss aber Beachtung auf zwei Ebenen finden. Da die grundlegenden Wahrheiten des christlichen Glaubens von Anfang an in Metaphern ausgedrückt worden sind, müssen sie methodisch als Metapher gelesen und untersucht werden. Auf der anderen Seite hat die Metapher eine Kraft der Überzeugung und der Begeisterung, die die spekulative Sprache oft nicht hervorrufen kann. Die Metapher ist also nicht nur ein Mittel für die Pastoral und Verkündigung, sondern sie hat auch ihren festen Ort in der systematischen Theologie. ${ }^{7}$ Man kann hier dem Postulat Nietzsches nachgehen, der in Jenseits von Gut und Böse fordert: „Je abstrakter die Wahrheit ist, die du lehren willst, um so mehr musst du noch die Sinne zu ihr verführen. ${ }^{\text {8 }}$ Im Folgenden soll daher anhand der Interpretationen von Nietzsches Werk bei Krzysztof Michalski das Potenzial der Metapher zur Beschreibung der Wirklichkeit dargestellt und für die Theologie fruchtbar gemacht werden.

6 Vgl.P. Liszka, Metafora w Objawieniu i w teologii, S. I-4, 8-Io, http://www.piotrliszka.strefa.pl/txt/r8metafora\%2or.pdf (II.I2.20I6).

7 Mit dem Phänomen der Metapher in der Theologie beschäftigt sich in seiner Monographie: J. Hartl, Metaphorische Theologie. Grammatik, Pragmatik und Wahrheitsgehalt religiöser Sprache, Berlin 2008.

8 F. Nietzsche, Jenseits von Gut und Böse, München 1999, S. 95 (Kritische Studienausgabe, 5). 


\section{Gegenwärtige Bedeutung Nietzsches in den Augen von Michalski}

Seit den 9oer Jahren wendete sich Krzysztof Michalski, der Warschauer Philosophieprofessor und Gründer des Instituts für die Wissenschaften vom Menschen in Wien, dem Werk von Friedrich Nietzsche zu. ${ }^{9}$ Die Arbeit mündete 2007 in seinem Buch zum Denken Nietzsches, The Flame of Eternity.

Das Interesse Michalskis für Friedrich Nietzsche gründete in seiner Überzeugung, dass Nietzsche wie kaum ein anderer Autor eine besondere Bedeutung für das Verständnis der heutigen Zeit haben könnte. Er kann uns nach Michalski in zwei wichtigen Aspekten helfen. Erstens sei Nietzsche der Autor, der die fundamentalen Begriffe unserer philosophischen Tradition wie Wahrheit, das Gute, das Schöne oder Gott am besten auf die heutige Erfahrung übertragen kann:

(...) die Erfahrung einer nicht besiegbaren Verschiedenheit der Welt, also die tief in unserem Bewusstsein liegende Überzeugung, dass alle Perspektiven, Gesichtspunkte, all die Fragmente, in die sich die Welt im Leben des heutigen, westlichen Menschen zerschlägt, sich nicht in eine Einheit zusammensetzen (und sie müssen es auch nicht). Wie ist über die Wahrheit, Gott, das Schöne oder Gute in der Welt zu denken, in der die Unterschiede auf keinen gemeinsamen Grund verweisen, nichts voraussetzen, was gemeinsam wäre. ${ }^{\text {IO }}$

Auf der anderen Seite könne uns Nietzsche eine Hilfe sein, um zu verstehen, was die Feststellung der Gottesexistenz für den heutigen Menschen bedeuten kann. ${ }^{\text {II }}$ Dadurch versucht Michalski auch einen neuen Zugang zur Religion in der Gegenwart zu öffnen:

9 I993 hält Michalski zum ersten Mal ein Seminar über Nietzsche an der Boston University und im darauffolgenden Jahr an der Universität Warschau. Ab diesem Zeitpunkt beschäftigt er sich kontinuierlich mit Nietzsche, hält zahlreiche Seminare und Gastvorträge zur Philosophie Nietzsches. Vgl. z.B. Iwm-Newsletter 4I (Juli-September 1993), S. 8 und IwM-Newsletter 44 (März-April I994), S. 9.

Io K. Michalski Interview mit Karolina Wigura, Nietzsche nasz wspótczesny, Dziennik (Europa supplement), 3-4.01.2009, S. 15. e.Ü.

II Vgl. K. Michalski, Nietzsche nasz wspótczesny, S. I5. 
Das Schaffen Nietzsches kann man nicht ohne Recht als ,Ringen mit Gott' bezeichnen. (...) Aber vielleicht (...) die radikale Kritik, der Nietzsche unsere Begriffe und unser Wissen unterzieht, öffnet auch einen Weg zum Verständnis der Religion, das auch für eine Vernunft überzeugend sein kann, die in ihrer Vielfallt der Sinnesangebote, Kulturen und der durch Wissenschaft durchdrungenen Gegenwart lebt. ${ }^{\text {I2 }}$

\section{I. Michalskis Zugang zur Sprache}

Michalski beschäftigte sich nie ausdrücklich mit dem Phänomen der Sprache. Es lässt sich aber bei ihm in diesem Bereich ein Einfluss von Jan Patočka und Hans-Georg Gadamer feststellen. In seinem frühen Text: Sprache-Wirklichkeit-Vernunft kommt Michalski zu der Feststellung, dass die Sprache nicht nur ein Kommunikationsmittel ist, sondern die Art und Weise, wie die Welt erscheint. Sie hat eine mitgestaltende Funktion bei der Konstitution der Welt:

Die Sprache - und zwar die wirklich gesprochene, die Alltagssprache ist wie ein Feld, auf dem die Welt erscheint, wie sie ist, und wo sich erst unser Wissen über diese Welt bildet. Patočka nannte es die „phänomenale Sphäre“, die Sphäre des Erscheinens. (...) Die Sprache ist nicht etwas, das neben anderen Dingen erscheint. Sie ist eine Struktur des Erscheinens selbst. Es ist nicht wahr, daß die Welt für uns und für alle möglichen Subjekte erscheint, bevor wir zu sprechen beginnen. Die Welt erscheint, indem wir sprechen. Wenn man in diesem Sinne nach der Sprache fragt, fragt man danach, wie es überhaupt möglich ist, daß die Welt erscheint und erscheinen kann. ${ }^{13}$

Der erscheinenden Welt versuchen wir immer einen Sinn zu geben. Dieser kann aber - und hier stützt sich Michalski auf Gadamer - nur in einem Prozess, im Dialog mit Kultur, Tradition und dem eigenen Leben entdeckt werden. Wir erkennen - auch uns selbst - indem wir leben, in der Lebenspraxis. Wir nehmen Teil an einem Sinn, der ständig im Werden ist und der im Dialog entsteht:

I2 K. Michalski, Ptomień wieczności. Eseje o myślach Fryderyka Nietzschego, Kraków 2007, S. I3. e.Ü.

I3 K. Michalski, Sprache-Wirklichkeit-Vernunft, in: E. Busek, M. Peterlik [Hrsg.], Sprache und Phantasie. Ein Gespräch zwischen Wissenschaft und Politik, Wien 1984, S. I8. 
Der Sinn, der in Geschichte beinhaltet ist, in der Tradition, in der Kultur, ist selbst im ständigen Werden - d.h. er wird durch einen jeden Schritt von uns, jede Entscheidung, jede Erkenntnis bestimmt: kurzum, durch unsere Lebenspraxis. In jedem Akt unseres Lebens ist der Sinn des Ganzen gegenwärtig, das das bestimmt, was wir sind. Gleichzeitig konstituiert sich dieser Sinn erst dadurch und kein Akt kann als Abschluss dieses Prozesses gelten. Genauso wie keiner ist sein Anfang. ${ }^{14}$

Nach Michalski sollten die Worte Gadamers als ein direkt zu mir ausgerichtetes Wort verstanden werden, als ein Argument in der Diskussion, die immer vom Neuem beginnt. Michalski zufolge kann man Gadamer mit Nietzsche vergleichen, dessen Werk ein lebendiger Prozess ist. Es geht nicht darum, dass er uns etwas Handfestes zu bieten hat, sondern dass er uns in eine Debatte einbezieht. ${ }^{15}$ Diese Debatte muss von uns mitgestaltet werden. Es reicht also nicht, dass wir mit konkreten Begriffen die Elemente unserer Wirklichkeit einer festgemachten Theorie zuordnen, sondern die Argumente müssen durch unser Leben getragen werden. Dieses Verständnis zieht sich durch die ganze Interpretation des Werkes Nietzsches von Michalski und sie führt uns zur Erklärung, warum Nietzsche sich so oft des Mittels der Metaphern bedient.

\subsection{Metapher als Ausdrucksmittel DER Wichtigsten Gedanken}

Die Wörter, Argumente und Metaphern, die wir bei Nietzsche finden, können nach Michalski nicht einfach als eine philosophische Argumentationskette verstanden werden. Nietzsche wolle uns in eine Debatte einbeziehen, die uns etwas angeht. Die Sprache, der er sich bedient, muss auf uns eine Wirkung haben:

Sie wollen verletzen oder Begeisterung wecken, Widerspruch oder Solidarität ernten. Denn nur Wörter, die begeistern oder verletzen, so Nietzsche, lassen sich auch verstehen. Die einzige Möglichkeit uns zu überzeugen, ist für Nietzsche, uns in den Streit hineinzuziehen, der uns unmittelbar angeht oder besser, den Beweis zu erbringen, dass wir,

I4 K. Michalski, Zrozumieć przemijanie, Warszawa 20II, S. 282-283. e.Ü.

I5 Vgl. K. Michalski, Zrozumieć przemijanie, S. 250, $26 \mathrm{I}$. 
ob wir wollen und darum wissen oder nicht, längst in diesen Streit eingebunden sind $(\ldots)^{\mathrm{I} 6}$

Die Funktion der Metapher reicht jedoch viel tiefer und beschränkt sich nicht nur auf ihre Wirkung. Der zweite Aspekt der Metapher hängt mit der bekannten nietzscheanischen Spannung zwischen Wissen und Leben zusammen. In der Interpretation dieser Spannung durch Michalski handelt es sich grob gesagt darum, dass das Leben nicht von meinem Wissen unabhängig sein kann (und umgekehrt). Einerseits hat das Leben immer schon eine Seite, die ich verstanden habe, die ich mit einem Netz von Bedeutungen bedecke. Anderseits aber ist das Leben immer mehr als das, was ich schon weiß oder verstehe. ${ }^{\text {t7 }}$ Es zeigt sich dabei, dass die Begriffe nie das Leben fassen können, dass sie zerbrechlich sind. Wenn das Wissen seine Schwäche auch offenbart, affirmiert es gleichzeitig das Leben. Es geht nicht um konkrete Inhalte, sondern um ein Leben, das alle Begriffe übersteigt, ,als ungeahnte, hinter den Begriffen verborgene Wirklichkeit (...), als Neuangang, als Schaffen. Als Chance auf Freiraum, auf weites Feld. “'

Das Leben lässt sich mit keinen Begriffen fassen und das Wissen wiederum beinhaltet eine eigene Spannung. Dies versucht Nietzsche mit Hilfe der Metaphern wiederzugeben. Die Metaphern braucht er hier, denn die Worte „reichen nicht aus, meint Nietzsche, Worte sagen, was ist, was wie ist, dieses so, jenes etwas anders." ${ }^{19}$

Michalski formuliert die Gedanken im Kontext der ewigen Wiederkehr des Gleichen, also dem wichtigsten Gedanken Nietzsches. Der Grundgedanke lässt sich also nicht in Worte fassen, nicht begreifen. Um den Gedanken zu beschreiben, bedarf Nietzsche des Mittels der Metapher:

Metaphern beziehen Worte auf etwas außerhalb ihrer Bedeutungen, verweisen auf ein Mehr an Sinn, das in ihnen verborgen liegt. Diese Funktion kommt auch den Worten in der Zarathustra-Geschichte zu:

I6 K. Michalski, Flamme der Ewigkeit, über. T. Weiler, Unveröffentlichte Übersetzung im Michalski-Archiv am Institut für die Wissenschaften vom Menschen in Wien, S. 29.

I7 Vgl. K. Michalski, Flamme, S. 4I.

I8 K. Michalski, Flamme, S. I39.

I9 K. Michalski, Flamme, S. I40. 
Sie bedeuten nicht nur, was sie bedeuten, sondern verweisen durch das metaphorische Mehr an Bedeutung auch auf die Situation, in der sie gesagt werden, auf etwas, das geschieht, auf etwas, das jemand tut. ${ }^{20}$

Die Metaphern sind stärker als jede Interpretation, sie zeigen, dass es noch eine andere, weitere Realität gibt. ${ }^{21}$ Die Metaphern dienen Nietzsche nicht nur als Schilderung eines Phänomens, sondern sie werden - nach Michalski - zu Metabegriffen, die immer auf etwas anderes, größeres, offenes, auf eine Grenze unseres Wissens verweisen. ${ }^{22}$

Dieses Verständnis der Metapher übernimmt Michalski für sein eigenes Werk. In einem Fernsehinterview bekennt er, dass die Frage, die ihn seit seiner Studienzeit beschäftigt, das Problem der Zeit sei. ${ }^{23}$ Ein grober Überblickt über seine Werke bestätigt diese Aussage. Seine Philosophie kreist immer um die stark miteinander verknüpften Themen: die Zeit, das Werden und die Vergänglichkeit. Bei den Versuchen, diese Phänomene zu verstehen, sucht Michalski Hilfe in der philosophischen Tradition. Trotz der intensiven Auseinandersetzung mit Heidegger und Husserl kann er diese Themen spekulativ nicht ausdrücken. Letztendlich bedient er sich in seinem Werk zur Beschreibung der Zeit, des Werdens und der Vergänglichkeit der Metapher, die für ihn die einzige Möglichkeit darstellt, sich diesen Phänomenen anzunähern. Die Zeit kann nach Michalski nur als spielendes Kind, als Fluss, als Wind oder Feuer, als die ewige Wiederkehr dargestellt werden.

\section{ZuSAmMENFASSUNG}

Die Geschichte Zarathustras knüpft von Anfang bis zum Ende an die Geschichte Jesu an. Die Erzählung von Zarathustra lebt von biblischen Bildern, Begriffen und Metaphern. Für Michalski ist diese Geschichte nicht nur eine Kritik am Christentum, sondern er sieht gewisse Ähnlichkeiten zu Evangelien und Paulusbriefen. ${ }^{24}$ Auch wenn jetzt nicht

20 K. Michalski, Flamme, S. I4I.

2I Vgl. K. Michalski, Flamme, S. 87.

22 Vgl. K. Michalski, Flamme, S. I4I.

23 Vgl. TVp Kultura - Rozmowy istotne, odc. 83. Ein Fernsehinterview von Agata Bielik Robson mit Krzysztof Michalski, http://vod.tvp.p1/176813/krzysztofmichalski?vdp=ıo (II.I2.20I6).

24 Vgl. K. Michalski, Flamme, S. III, I46. 
dem Gespräch zwischen Paulus (oder dem Autor der Apokalypse) ${ }^{25}$ und Nietzsche nachgegangen werden kann, ist die Ähnlichkeit, die sich zuerst auf der sprachlichen Ebene feststellen lässt, bei allen drei $\mathrm{Au}-$ toren - Paulus, Nietzsche und Michalski - der Rückgriff auf die Metapher, wenn man über die wichtigsten Inhalte der eigenen Botschaft spricht. Man darf selbstverständlich die Inhalte nicht auf Metaphern reduzieren, aber es ist trotzdem bemerkenswert, dass man die - mit Nietzsche gesprochen - „abgründigsten Gedanken“ nicht mit Hilfe der eigentlichen Begriffe vermitteln kann.

Natürlich kann das Mittel der Metapher das Problem der Krise der theologischen Sprache, die im größeren Kontext der Gotteskrise steht, nicht lösen. Die Verwendung der Metapher, die ganz in der Tradition der christlichen Theologie steht, kann aber zwei wichtige Funktionen haben: Sie dient nicht nur der Beschreibung der Wirklichkeit, sondern hat eine Wirkung auf den Empfänger der Botschaft. Darüber hinaus kann sie zeigen, dass bei den wichtigsten Gedanken die Begriffe zerbrechen. Wenn man dann diese Gedanken mit Hilfe der Metapher darzustellen versucht, können sie nicht nur leichter zugänglich sein, sondern schon durch die Form weisen sie darauf hin, dass sie in sich eine Offenheit haben, die sich nicht bis zum Ende begreifen lässt. Mit anderen Worten: Sie verweisen auf etwas Größeres, die Wirklichkeit Übersteigerndes. Es scheint, dass die Metapher ein geeignetes - selbstverständlich nicht das einzige Mittel - zur Reflexion über die biblische Offenbarung darstellt, die schließlich nicht nur Informationen über Gott bietet, sondern vor allem auf unsere Existenz abzielt und uns zu einer Gemeinschaft mit Gott führen will. Darin spiegelt sich aber auch eine allgemeine Eigenschaft unserer Sprache: „Wie der wörtliche Sinn, indem er sich durch seine Unstimmigkeit selbst zerstört, einem metaphorischen Sinn den Weg bahnt, so setzt die wörtliche Referenz, indem sie aufgrund ihrer Unangemessenheit zusammenbricht, eine metaphorische Referenz frei, durch die die dichterische Sprache zwar nicht sagt, ,was ist', doch ,wie' die betrachteten Dinge sind. ${ }^{\text {"26 }}$

25 Vgl. K. Michalski, Flamme, S. 50.

26 P. Ricoeur, Die lebendige Metapher, München 1986, vir. Zitiert nach: A. Tebartz-van Elst, Ästhetik der Metapher. Zum Streit zwischen Philosophie und Rhetorik bei Friedrich Nietzsche, München 1994, S. 155 . 


\section{BibLIOGRAPHIE}

Hartl J., Metaphorische Theologie. Grammatik, Pragmatik und Wabrheitsgehalt religiöser Sprache, Berlin 2008.

Liszka P., Metafora w Objawieniu $i$ w teologii, http://www.piotr-liszka.strefa. $\mathrm{pl} / \mathrm{txt} / \mathrm{r} 8$ metafora\%2OI.pdf (II.I2.20I6).

Metz J. B., Kirche in der Gotteskrise, in: C. Amery [Hrsg.], Sind die Kirchen am Ende?, Regensburg 1995, S. 158-I75.

Michalski K. Interview mit Karolina Wigura, Nietzsche nasz wspótczesny, Dziennik (Europa supplement), 3-4.01.2009, S. 15.

Michalski K., Flamme der Ewigkeit, über. T. Weiler, Unveröffentlichte Übersetzung im Michalski-Archiv am Institut für die Wissenschaften vom Menschen in Wien.

Michalski K., Ptomień wieczności. Eseje o myślach Fryderyka Nietzschego, Kraków 2007.

Michalski K., Sprache-Wirklichkeit-Vernunft, in: E. Busek, M. Peterlik [Hrsg.], Sprache und Phantasie. Ein Gespräch zwischen Wissenschaft und Politik, Wien I984, S. I4-I8.

Michalski K., Zrozumieć przemijanie, Warszawa 2011.

Peters T. R., Mehr als das Ganze. Nachdenken über Gott an den Grenzen der Moderne, Ostfildern 2008.

Ricoeur P., Die lebendige Metapher, München 1986.

Tebartz-van Elst A., Ästhetik der Metapher. Zum Streit zwischen Philosophie und Rhetorik bei Friedrich Nietzsche, München 1994.

Weidmann F., Sprache und Religionsunterricht, in: F. Weidmann (Hrsg.), Didaktik des Religionsunterrichts. Ein Leiffaden, Donauwörth 1997', S. I64-I79. 